\title{
BMJ Open Rationale, design and protocol of a cross-sectional study on pregnancy- related cardiovascular diseases in Tanzania (PRECARDT): burden, characterisation and prognostic significance at delivery
}

Abel Makubi, ${ }^{1,2}$ Pilly Chillo (D) , ${ }^{1}$ Reuben Mutagaywa, ${ }^{1}$ Belinda Balandya, ${ }^{1}$ Peter Kisenge, ${ }^{3}$ Vincent Tarimo, ${ }^{4}$ Eva Mujuni, ${ }^{5}$ Evarist B Msaki, ${ }^{2}$ Josephine Mgaya, ${ }^{1}$ Albert Kihunrwa, ${ }^{2,5}$ Mohamed Janabi, ${ }^{3}$ Gideon Kwesigabo, ${ }^{1}$ Julie Makani, ${ }^{1}$ Lindsay Kendall, ${ }^{6}$ Juliet Addo, ${ }^{6}$ Bruno Mmbando, ${ }^{7}$ Karen Sliwa ${ }^{8}$

To cite: Makubi A, Chillo P, Mutagaywa $\mathrm{R}$, et al. Rationale, design and protocol of a crosssectional study on pregnancyrelated cardiovascular diseases in Tanzania (PRECARDT): burden, characterisation and prognostic significance at delivery. BMJ Open 2021;11:e049979. doi:10.1136/ bmjopen-2021-049979

\section{- Prepublication history for} this paper is available online. To view these files, please visit the journal online (http://dx.doi. org/10.1136/bmjopen-2021049979).

Received 11 February 2021 Accepted 07 December 2021

Check for updates

(C) Author(s) (or their employer(s)) 2021. Re-use permitted under CC BY-NC. No commercial re-use. See rights and permissions. Published by BMJ.

For numbered affiliations see end of article.

Correspondence to

Dr Abel Makubi;

abelmakubi@gmail.com

\section{ABSTRACT}

Introduction The paucity of data describing cardiovascular disease (CVD) in pregnancy in many parts of Africa including Tanzania has given rise to challenges in proper management by the healthcare providers. This study is set out to (1) determine the prevalence of a range of CVDs during pregnancy in women attending antenatal clinics in Tanzania and (2) determine the impact of these CVDs on maternal and fetal outcomes at delivery.

Methods and analysis This is a cross-sectional study with a prospective component to be conducted in two referral hospitals in Tanzania. Pregnant women aged $\geq 18$ years diagnosed with a CVD during the antenatal period are being identified and extensively characterised by performing clinical assessment, modified WHO staging, electrocardiography, echocardiography and laboratory tests. Patients identified with CVDs (exposed) and a subset without (unexposed) will be followed up to determine maternal and fetal outcomes at delivery. A minimum sample of 1560 will be sufficient to estimate the prevalence of CVDs with a $95 \% \mathrm{Cl}$ of $2.75 \%$ to $5.25 \%$. Ethics and dissemination The study is being conducted in accordance with the Helsinki declaration on studies involving human subjects. Ethical approvals have been obtained from Muhimbili University (reference number DA.282/298/01.C/) and Bugando Medical Centre (reference number CREC/330/2019) Ethics Committees. Informed consent is sought from all potential participants before any interview or investigations are performed. Study findings will be disseminated to the scientific community through different methods. Results will also be communicated to policymakers and to the public, as appropriate.

\section{INTRODUCTION}

The magnitude of cardiovascular diseases (CVDs) during pregnancy is increasing across the world, resulting in significant morbidity and mortality. ${ }^{1-3}$ In high-income countries
Strengths and limitations of this study

To the best of our knowledge, this will be the first study conducted in a large population of pregnant women in Tanzania to determine the prevalence of cardiovascular diseases (CVDs) and the effect on maternal and fetal outcomes at delivery.

- The extensive assessment of CVDs using clinical assessment and different imaging modalities will potentially capture even asymptomatic CVDs.

- The prospective follow-up of all pregnant women from antenatal visits though delivery and the systematic assessments of the mother and fetus will reduce bias in the outcome of interest.

- The extensive data generated at enrolment have the potential for future long-term follow-up research to unravel other yet unknown risk factors for maternal and fetal outcomes, including genetic studies.

- A potential limitation of this study is the identification of maternal and fetal outcomes only at delivery, thereby potentially missing some outcomes that may manifest later.

(HIC), CVDs are seen in $0.2 \%-4 \%$ of the pregnant women. ${ }^{4}$ In contrast, the prevalence of these conditions is largely unknown in Tanzania and many parts of Africa. Aetiology and outcome of CVDs vary between low income and HIC. ${ }^{16}$ In both populations, women may have pre-existing disease unmasked by pregnancy (eg, mitral stenosis due to rheumatic heart disease (RHD)), a superimposed condition unique to pregnancy (eg, peripartum cardiomyopathy) or a complication of pregnancy itself (eg, hypertensive disorders of pregnancy). 
In HIC, the increasing trend of CVDs in pregnancy has been reported to be associated with a number of factors, including a shift of childbearing age towards older women who are more likely to present with CVDs of ageing and lifestyle. ${ }^{7}$ Also, as a result of better management and increased survival of patients with congenital heart disease (CHD), there has been an increased number of pregnant women with repaired or monitored CHD. ${ }^{8}$ In the UK, for example, cardiac diseases have become the leading cause of indirect maternal death, occurring at a rate of 2.1 per 100000 pregnancies. ${ }^{9}$ Ischaemic heart diseases (24\%), cardiomyopathies (27\%), sudden arrhythmic cardiac deaths $(18 \%)$ and aortic dissection $(11 \%)$ are the primary cardiac diseases in the UK, with RHD still a frequent cause of maternal death $(7 \%)$, particularly in immigrants. ${ }^{9}$

Continued lack of awareness about maternal CVDs in Africa often leads to suboptimal care and missed opportunities during pregnancy and after delivery. ${ }^{10}$ During the past decade, a steady increase in institutional maternal mortality rate for cardiac disease in Africa has been reported. ${ }^{112}$ In South Africa, after non-pregnancy-related infections, cardiac disease is the second most common cause of indirect maternal death, with postpartum cardiomyopathy and complications due to RHD contributing to more than $50 \%$ of the cases. ${ }^{112}$ Similar data are very limited in other parts of Africa. In Tanzania, there are no studies reporting on the magnitude of CVDs in this population as well as data on prognosis. As embodied in the Millennium Development Goals and later the Sustainable Development Goals, the death of a mother not only affects the survival of the immediate maternal period but also has effects throughout the life course of the child as well as the next generation. ${ }^{13} 14$

A number of registries and contemporary analyses of pregnancy outcomes of women with heart diseases have been reported in the literature (table 1). Of note, more recent results from the large EURObservational Research Programme Registry of Pregnancy and Cardiac disease registry have added to our understanding of the maternal and fetal outcomes of women with different cardiac conditions, including women with uncorrected $\mathrm{CHD},{ }^{15}$ transposition of great arteries, ${ }^{16}$ aortic coarctation ${ }^{17}$ and in those with ischaemic heart disease. ${ }^{18}$ Most of these studies are predominantly from HIC, and many have focused on women with $\mathrm{CHD}^{15-1719-21}$ as well as in general CVDs. ${ }^{2} 3182223$ Although the majority of studies have reported an increased risk of adverse maternal and fetal outcomes, ${ }^{3} 15192022-26$ others did not show this increased risk. ${ }^{21} 27$ In Australia, Sartain et al found RHD to have impact on maternal cardiac outcomes only in those with high predictable risks, and they report no maternal or fetal mortality in their cohort of 54 pregnant women with RHD. ${ }^{27}$ However, RHD was associated with an increased maternal and fetal adverse outcomes in studies from Egypt $^{26}$ and China. ${ }^{28}$ In South Africa, Sliwa $e t a l^{29}$ showed a disease pattern markedly different to that seen in HIC with RHD, cardiomyopathies, coronary heart disease and some previously not diagnosed CHD as being major problems, often complicated by HIV/AIDS as a comorbidity. The data also demonstrated a significantly higher maternal mortality rate $(5.9 \%)$ among pregnant women with CVDs compared with $1 \%$ in a study of a similar population conducted in Europe. ${ }^{2}$

CVDs in pregnancy have not been well studied and characterised in Africa. Availability of more data and establishment of regional registries from this continent aim to accumulate evidence for the development of locally acceptable treatment guidelines and possible reduction of maternal morbidity and mortality, resulting in better fetal outcome.

\section{Study rationale}

The burden, characteristics and short-term prognosis of CVDs on pregnancy have been fairly studied in HIC but considerably less in developing countries and are largely unexplored in many parts of Sub-Saharan Africa, including Tanzania. This study is expected to contribute to our understanding of the magnitude and possible uniqueness of the clinical patterns of CVDs in pregnant women in Tanzania, and their short-term prognosis. Such knowledge is essential in the prevention and treatment of CVDs in pregnancy. The data generated may also provide evidence for the development of locally acceptable management guidelines.

The study may also create awareness for active screening of these CVDs in pregnancy and possibly lead to improvement in maternal and fetal outcomes at delivery. In addition, targeting the risk factors associated with poor outcomes will have a positive impact on preventive measures.

Finally, the study will lay a platform for establishing a national/regional maternal CVDs registry in Tanzania and East Africa, in which evidence-based treatments/practices can be agreed on, implemented, and the natural history of these conditions is closely monitored.

\section{Research questions}

1. How common are CVDs among pregnant women in Tanzania?

2. Are the clinical presentation of pregnant women with CVD different from those women without CVD?

3. Are maternal and fetal outcomes at delivery worse for pregnant women with CVD compared with those without CVD?

\section{Study objectives}

The primary objectives of this study are:

1. To determine the prevalence of CVDs among pregnant women visiting their antenatal clinics (ANC) in Tanzania.

2. To describe (as determined by clinical, electrocardiographic, echocardiographic parameters and WHO risk stratification) the types of CVDs observed in pregnant women visiting their ANC in Tanzania. 
Table 1 Examples of studies with reports on maternal and fetal outcome in pregnant women with heart diseases

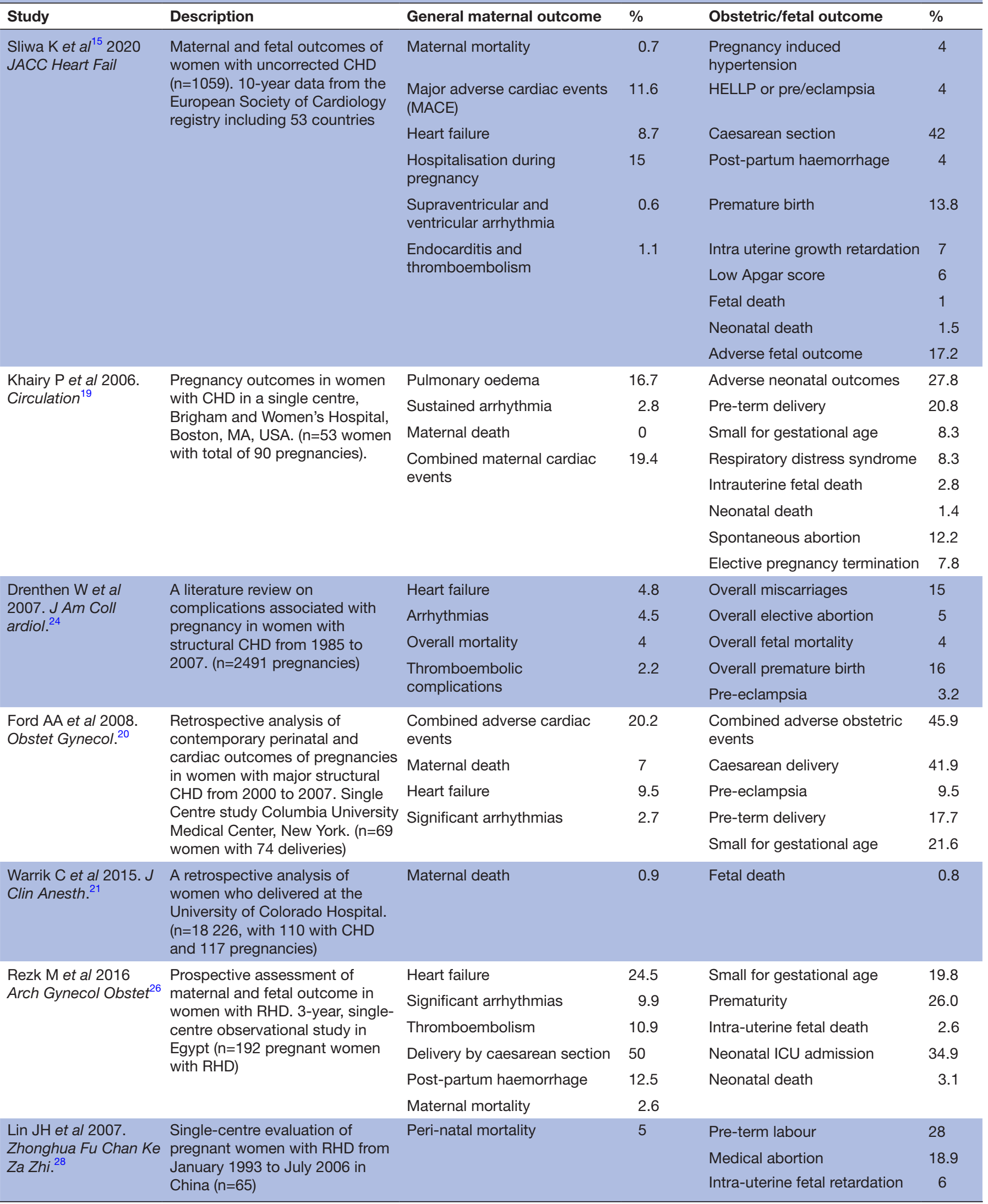




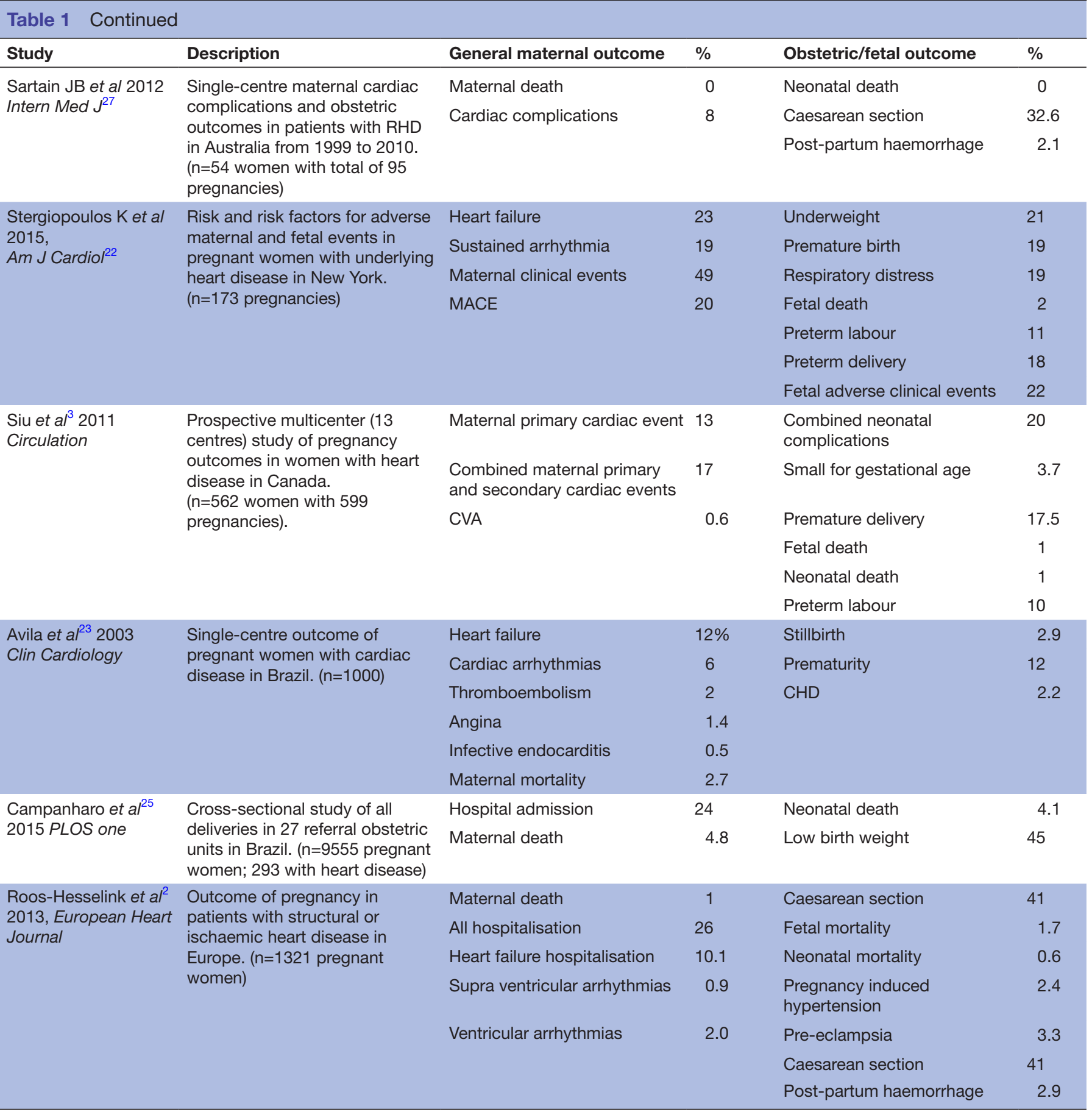

CHD, congenital heart disease; HELLP, Hemolysis, Elevated Liver enzymes and Low Platelets; RHD, rheumatic heart disease.

The secondary objectives are:

1. To compare the incidence of a composite of maternal adverse clinical outcomes (MACO) (all cause death, mode of delivery-caesarean section, heart failure, eclampsia and obstetric haemorrhage requiring admission) in pregnant women presenting with CVDs versus without CVDs at their ANC and followed-up prospectively to delivery.

2. To compare the incidence of a composite of fetal adverse clinical events (FACE) (fetal death, prematurity, intrauterine fetal growth retardation, birth weight and respiratory distress) at delivery in pregnant women presenting with CVDs versus without CVDs (as diagnosed at baseline).

\section{METHODS AND ANALYSIS}

\section{Study design}

This will be a hospital-based cross-sectional study with a prospective component to be conducted in two centres 


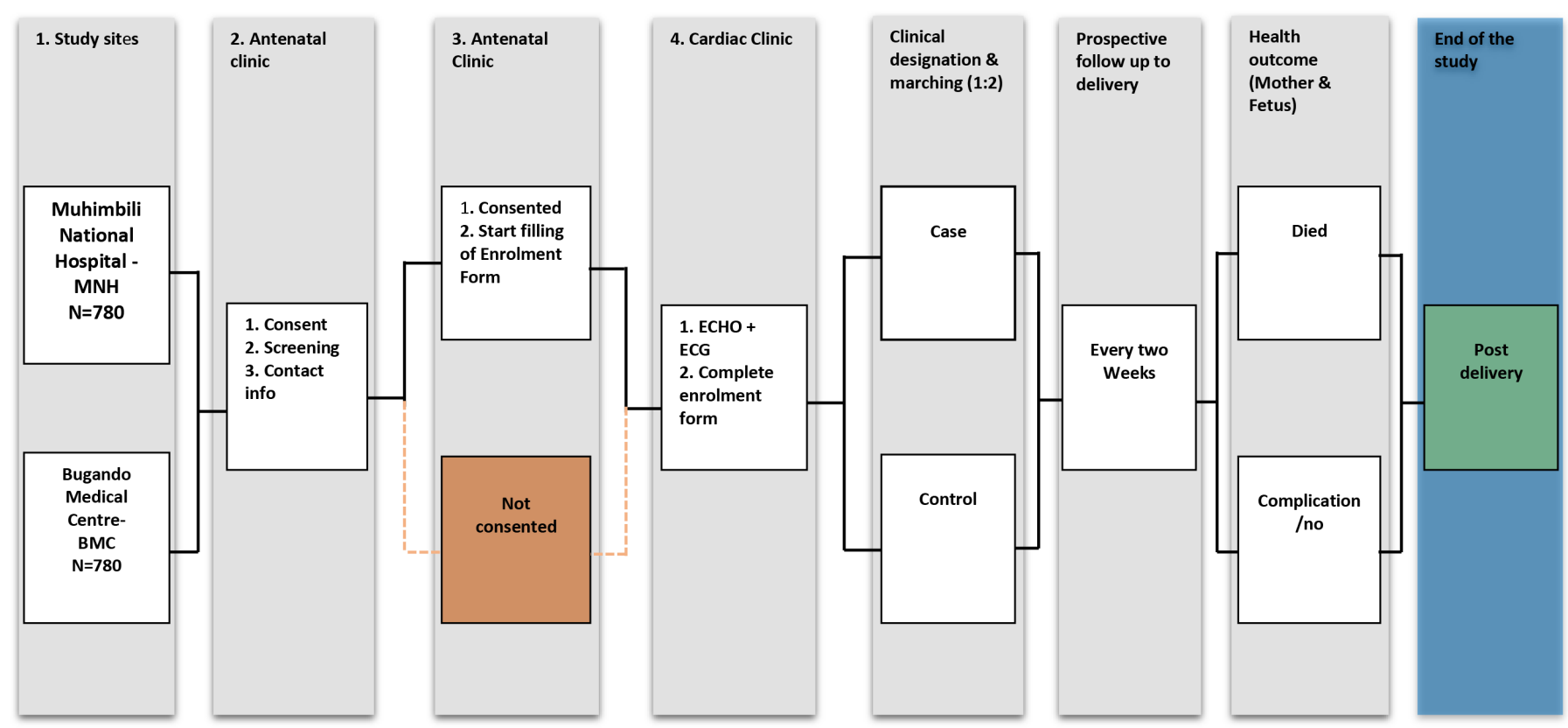

Figure 1 Study design of cross-sectional and prospective components. BMC, Bugando Medical Centre; ECHO, echocardiogram.

as illustrated in figure 1. The two sites are selected to be representative of the urban and rural populations in Tanzania.

\section{Study setting, antenatal and CVDs services in Tanzania}

The study will be conducted in the ANC and Cardiac units of the two main referral Hospitals in Tanzania, namely, Muhimbili National Hospital in Dar es Salaam and Bugando Medical Centre in Mwanza located at a distance of over $1100 \mathrm{~km}$ (from Dar es Salaam). Dar es Salaam is composed of urban diverse ethnic groups compared with Mwanza, a cosmopolitan city with mixture of different ethnic rural groups.

Muhimbili National Hospital is a national and teaching hospital located in the largest City of the Country, Dar es Salaam region, serving mainly the urban and the referred populations. The hospital has ANC services and is closely linked to cardiology services at Jakaya Kikwete Cardiac Institute, within the same campus. An average of 80 pregnant women attend the clinic daily, and $80 \%$ of them attend in view of their current pregnancy while $20 \%$ are post-natal follow-ups.

Bugando Medical Centre is the second referral consultant and University Teaching Hospital in Tanzania. It is a referral centre for tertiary specialist care for eight regions and serves a catchment population of approximately 18 million people. It mainly serves a rural population. The hospital has both antenatal clinic and cardiology clinics. About 80 pregnant women attend the clinic daily, which is run on 5 days a week. At each clinic, up to 30 pregnant women per day are attending for their first time.
As per National guidelines, pregnant women in Tanzania are required to start ANC visits as soon as possible in the first trimester of pregnancy (though many of them come at different stages of pregnancy) and are followed-up to delivery.

Study duration

The first participant was enrolled in October 2019 and we expect the follow-up of the last enrolled participant to end in May 2021.

The inclusion and exclusion criteria are presented in table 2.

\section{Power calculations and sample size}

Assuming the prevalence of CVDs in pregnant women to be as high as the $4 \%$ reported in $\mathrm{HIC}^{45}$ then a sample size of 945 will be sufficient to estimate the prevalence of CVDs with a $95 \%$ CI of $2.75 \%$ to $5.25 \%$. Recruitment

Table 2 Study population, inclusion and exclusion criteria

\begin{tabular}{ll}
\hline Inclusion criteria & Exclusion criteria \\
\hline $\begin{array}{l}\text { Pregnant women } \\
\text { attending the ANC }\end{array}$ & $\begin{array}{l}\text { Women not residing in Dar es } \\
\text { salaam or Mwanza }\end{array}$ \\
Age $\geq 18$ years & $\begin{array}{l}\text { Women with known diabetes, sickle } \\
\text { cell anaemia, asthma, coagulation } \\
\text { disorders, advanced HIV stage } \\
\text { (III-IV) and advanced renal failure } \\
\text { Refusal to give informed consent }\end{array}$
\end{tabular}

ANC, antenatal clinics. 
will be from one rural and one urban site, thus the prevalence of CVDs could vary by site and so to account for this, by assuming a design effect of $1.5 \%$ and $10 \%$ loss to follow-up, a minimum sample size of 1560 will be enrolled.

\section{Case definition}

1. A CVD event will include any/combinations of the following; known hypertensive or newly diagnosed hypertension, defined as systolic blood pressure $\geq 140$ $\mathrm{mm} \mathrm{Hg}$ and/or diastolic blood pressure $\geq 90 \mathrm{~mm} \mathrm{Hg}$ on three readings, known or newly diagnosed RHD, any other valvular disease, CHD, known or newly diagnosed cardiomyopathies, coronary heart disease, heart failure, pericardial disease (ie, tuberculosis), arrhythmias-atrial fibrillation/flutter, ventricular fibrillation and bundle branch block (2nd-3rd degree).

2. Heart failure as per European Society of Cardiology (ESC) diagnostic criteria. ${ }^{30}$

\section{Primary endpoints}

Prevalence of CVDs in pregnant women visiting ANC in Tanzania

\section{Secondary endpoints}

1. Incidence of a composite of MACO, defined as at least one of the followings: all cause deaths, heart failure, caesarean mode of delivery, eclampsia and obstetric haemorrhage requiring admission in pregnant women.

2. Incidence of a composite of FACE, defined as at least one of the followings: fetal death, prematurity, low birth weight and respiratory distress in the baby.

\section{Study protocol}

Initial assessment of pregnant women at the ANC

Every sampled pregnant woman $\geq 18$ years at any gestation age visiting the ANC will be given opportunity to consent her participation into the study, as shown in figure 2.
Clinical assessment and measurements (at the ANC)

At the ANC, all consenting eligible consecutive pregnant women will be interviewed and examined for sociodemographic and baseline clinical characteristics such as age, education level, occupation, blood group, history of any CVDs, smoking and alcohol consumption, chronic hypertension, HIV status, gestation age, parity, prior surgery or cardiac intervention, use of medication and known/ documented comorbidities such as renal disease, and diabetes.

\section{Cardiological examination}

At the cardiology clinic, detailed examination of the CVDs will include both ECG and an echocardiogram (ECHO) examination for all recruited women. Women with CVDs will be further stratified into four risk groups using a modified WHO risk classification for pregnant women with cardiac disease (figure 3). The WHO risk stratification tool is based on the disease severity and has a prediction value in the management of pregnant women. Depending on the diagnosis and severity of the disease, risk classification will range from class I (low risk) to class IV (contraindication for pregnancy), as used in the ESC Guidelines on the management of CVD during pregnancy. ${ }^{4}$

\section{Haematological evaluation and serum storage}

In all eligible pregnant women, blood will be collected for full blood count. The serum will be stored with the consent of participants, for up to 10 years after study completion for further tests (including genetic studies related to CVD or pregnancy) that may emerge in the future.

\section{Electrocardiography and echocardiography measurements}

A standard 12-lead ECG will be obtained in all eligible pregnant women referred to the cardiology unit from the ANC, being in supine position, in a quiet room, using

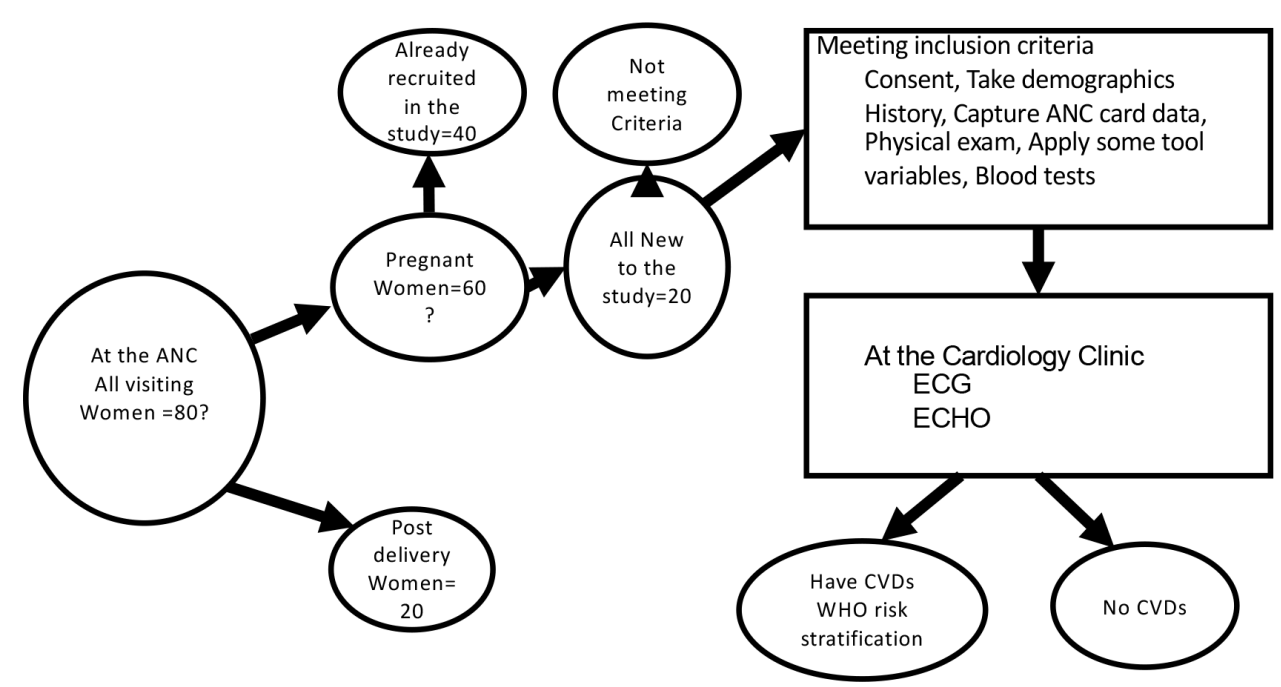

Figure 2 Weekly flow of pregnant women-identification and enrollment of participants. ANC, antenatal clinics; CVD, cardiovascular disease; ECHO, echocardiogram. 


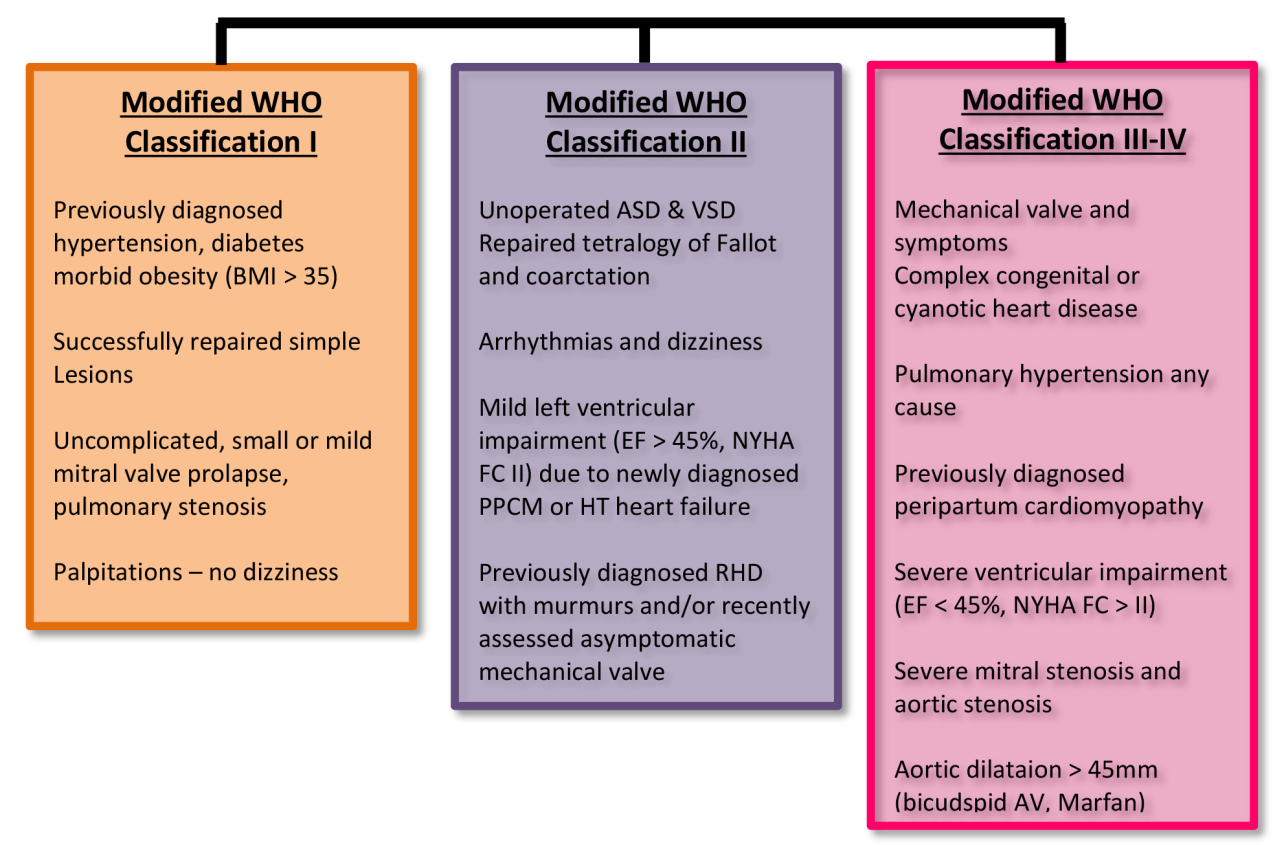

Figure 3 Modified WHO risk stratification. $\mathrm{RHD}$, rheumatic heart disease.

PHILIPS machine (Pager, Trim III). Recording will be done with standard ECG paper at a speed of $25 \mathrm{~mm} / \mathrm{s}$ and calibration of $10 \mathrm{~mm} / \mathrm{mV}$ standardisation.

Echocardiography will be initially performed in every pregnant woman prior to assigning into 'exposed/unexposed' groups using General Electric Vivid V.5 with a 2.5-5 MHz probe Trans-thoracic, M-mode, two-dimensional and tissue Doppler imaging (TDI) by an experienced echocardiographer. The focus will be on assessment of both the left ventricular dimensions and function. In particular, diastology will be an important aspect of the comprehensive echocardiographic assessment of these patients. Echocardiographic indices of diastolic dysfunction will include TDI, mitral diastolic flow profile and increased left atrial filling pressures. ${ }^{31}$ The ECG/ECHO findings will be interpreted by the author, reviewed and approved by a second senior and experienced cardiologist (A).

\section{Storage of ECG and ECHO data and independent evaluation of} interpretation

Data on ECG and ECHO will be stored in hard disks/ Compact Discs (CDs) in an access-controlled area, and it will be easily retrieved and backed up to allow an independent evaluation of $15 \%$ of the cases by another experienced cardiologist (B). In case of disagreement of the findings between (A) and (B), a third senior consultant cardiologist $(\mathrm{C})$ will be asked to review the ECHOs and provide the conclusion, taking into consideration both the clinical presentation and the ECHO findings.

\section{Inter observer variations in $\mathrm{ECHO}$ measurements}

As the ECHO machines are of similar General Electric (GE) models, the sonoechocardiographers will be trained on standardised image acquisitions, and we will perform monthly quality control for the two sites.
The follow-up sub-study

The study will involve follow-up of all pregnant women identified with CVDs versus a subset of those without, for MACO and FACE at delivery. Pregnant woman's CVD/ no-CVD status will be defined at enrolment, and any incident CVD during the follow-up period will not count for the purpose of assigning participants into the two groups. However, any incident CVD event will be documented on the case report forms (CRF).

\section{Selection of exposed patients}

All pregnant women identified with CVDs in the main study will be selected and considered as exposed patients.

\section{Selection and matching of a subset of unexposed participants}

Pregnant women identified without CVD events in the main study will form a pool of unexposed (figure 4). Matching by age ( \pm 5 years) and gestation age (rounded to 1 month) will be applied to identify the unexposed, two times the number of cases using simple random numbers generated using preset seeds. For each exposed case identified, a list of pregnant mothers meeting the criteria for matching with the index case will be prepared from which unexposed pregnant women will be selected. The unexposed participants will be selected if the list contains at least three times the number of each index exposed case, otherwise, unexposed women will be selected among the pool of unexposed individuals in the next clinical visit. Unexposed women (among those not yet delivered) will be selected immediately on identification of the corresponding exposed women.

Women whose pregnancies are terminated for any reason before delivery will have no further follow-up, but information on termination will be kept as an additional outcome measure. 


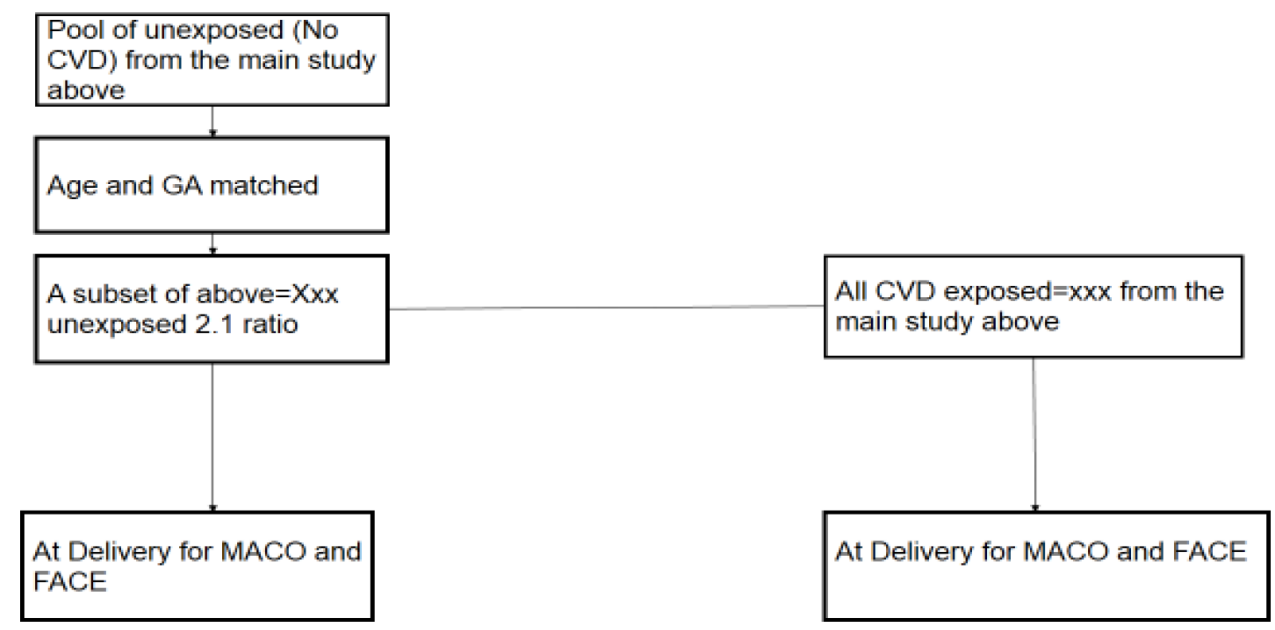

Figure 4 Identification of exposed and unexposed women and outcomes at delivery. CVD, cardiovascular disease; FACE, fetal adverse clinical event; MACE, major adverse cardiac event.

\section{Follow-up and assessment of secondary endpoints}

Clinical follow-up visits will be performed at delivery to establish any events of MACO and FACE (table 3). For participants who will not deliver at the recruiting hospital, follow-up data will be obtained by making phone contacts and physical follow-up (at the hospital of delivery) by a research nurse and study coordinators. Also, all enrolled mothers will be encouraged to receive their health services at the index hospital. In case of attendance elsewhere, they should have a phone number for the research clinician and nurse to send the message for any event, leading to birth (ie, labour).

\begin{tabular}{|c|c|c|c|}
\hline Variable & $\begin{array}{l}\text { At } \\
\text { enrolment }\end{array}$ & $\begin{array}{l}\text { During follow- } \\
\text { up in between }\end{array}$ & $\begin{array}{l}\text { At } \\
\text { delivery }\end{array}$ \\
\hline Informed consent & $x$ & $\begin{array}{l}\text { Passive } \\
\text { screening }-2 \\
\text { weekly by } \\
\text { a doctor or } \\
\text { woman on } \\
\text { demand }\end{array}$ & \\
\hline $\begin{array}{l}\text { Inclusion/exclusion } \\
\text { criteria }\end{array}$ & $x$ & & \\
\hline Demographics & $x$ & & \\
\hline Medical history & $x$ & $X$ & $X$ \\
\hline $\begin{array}{l}\text { Physical } \\
\text { examination }\end{array}$ & $x$ & & $x$ \\
\hline Medications & $x$ & & \\
\hline Full blood picture & $x$ & & \\
\hline $\mathrm{ECG}$ and $\mathrm{ECHO}$ & $x$ & & \\
\hline $\begin{array}{l}\text { Outcome events- } \\
\text { MACO and FACE }\end{array}$ & & & $X$ \\
\hline
\end{tabular}

To be collected in all exposed women (with CVDs) and in a subset of unexposed women (without CVDs).

CVD, cardiovascular disease; ECHO, echocardiogram; FACE, fetal adverse clinical event; MACE, major adverse cardiac event.
A research nurse will make monthly phone follow-up calls to all women with CVDs and a subset of those without (unexposed), until delivery to maintain a close contact with our team and determine whether there is any change in the expected date/place of delivery. Once mothers are admitted in labour, they will be encouraged to send message/calls to the research nurse. The research team will also arrange with the labour ward nurses to be contacted anytime a mother recruited into the study (determined by an identifiable study label on antenatal card) is admitted to the ward for delivery. Once contacted, the research nurse will follow-up the mother on the ward and precisely capture the maternal and fetal outcomes. Additionally, study nurses will visit the labour wards of the two hospitals daily to actively identify any study participant in labour.

\section{Handling loss to follow-up}

All pregnant women will be asked to provide their phone contacts as well as those of two close relatives/neighbours to ensure effective communication, including being unable to report for delivery at the primary hospitals.

\section{Data analysis plan}

Database management and statistical analyses will be performed with STATA software, V.13 and using the statistical software $R$ (https://cran.r-project.org/). Continuous data will be expressed as mean $\pm \mathrm{SD}$ if normally distributed or median with range/IQR if non-normally distributed. Comparison of means and proportions between subgroups at baseline will be performed using the Student-t, $\chi^{2}$ Fisher's or Wilcoxon rank-sum test statistics where appropriate. Categorical variables (primary and secondary) will be summarised using frequencies and percentages. Independent t test will be performed on secondary measures. The association between binary secondary response variable (eg, MACO) and independent predictors will be assessed using logistic regression model. The study secondary endpoints (MACO and FACE) will be taken as composite endpoints, and they will 
not be detailed for their separate diagnoses due to the limited sample size. Continuous variables will be assessed for normality assumptions, and where required, appropriate transformation will be done, and their association with independent predictors will be assessed using linear regression model. Interaction between independent variables will be explored during assessment of model adequacy. Statistical significance is defined as $p<0.05$. The analysis will follow a prespecified statistical analysis plan.

\section{Data management}

Data will first be captured on paper CRFs designed for the study, then entered into a specifically developed webbased (REDCap) platform for this project, at both sites. For quality control purposes, there will be double data entry with checks in $15 \%$ of the CRFs.

If a participant withdraws from the study, all medical record information related to her/him will be destroyed only if the participant asks that all information be withdrawn. If a participant does not request that all their information be destroyed, but to withdraw from future participation only, then any information collected prior to the withdrawal will be maintained. The principal investigator (PI) will be responsible for ensuring that the data collected are complete, accurate and recorded in a timely manner.

\section{Data security and confidentiality}

All consent forms will be kept in a locker (with a lock and Key) in the consent room. All patients will receive a study ID and the corresponding name only known to the site coordinator. The consent forms and CRFs will be collected by the site PI from the lockers to Muhimbili University (MUHAS) office where they will be stored. The exposed to the corresponding non-exposed will be linked through a unique ID number. The confidentiality of study participants will be protected by randomly assigning individual identifiers, which will be linkable to identifying information. All data will be anonymised.

\section{Ethical considerations}

Ethical approval has been obtained from the MUHAS (reference number DA.282/298/01.C/) and the Bugando Medical Centre (reference number CREC/330/2019) Ethics Committees. Informed consent (signed or thumbprinted) is being sought from all potential participants before any interviews or investigations are performed. Refusal to participate in the study does not, in any way, affect the management of the patient. Individuals who are not eligible for inclusion into the study are being referred back to their health facility for follow-up or are seen at the cardiac clinic as per normal protocols. Withdrawal from the study at any time lies within the participant's discretion and does not hinder the individual's right to appropriate healthcare. As the ANC is closely linked to cardiac clinic in the same hospital, women with CVDs are followed-up at the same cardiac clinic as per standard of care. Consent and ethical approval are sought from the study participants for storing the serum for 10 years after the study, or future tests (including genetic studies related to CVD or pregnancy). Personal and medical information identifying the participant (with the sample) are kept confidential.

\section{Plans for dissemination of research findings}

The findings will be disseminated in several forms (a) feedback research reports to the health facility sites and to the funder GlaxoSmithKline (GSK), (b) faculty symposium, research reports and journal club presentation in the participating hospitals, $(\mathrm{C})$ research reports to the Ministry of Health in Tanzania, (d) abstract presentation in local and international conferences, (e) university website announcement, (f) journal publications, (g) public release notification in media and research bulletin.

\section{Potential risks and mitigation plans}

Both ECG and ECHO investigations are non-invasive and carry no potential risk to the participants. During drawing of blood, participants may experience mild pain and/or swelling at the puncture site, and occasionally the puncture site could be infected. To minimise any risk, research nurse assistants have been trained on proper and sterile blood drawing procedures as well as trained to adhere to infection prevention and control procedures.

\section{Patients and public involvement}

Participants of this study gave their feedback regarding the data collection tools as part of their participation in the study. Feedbacks were given after data piloting

\section{Author affiliations}

${ }^{1}$ Muhimbili University of Health and Allied Sciences, Dar es Salaam, United Republic of Tanzania

${ }^{2}$ Bugando Medical Centre, Mwanza, United Republic of Tanzania

${ }^{3}$ Jakaya Kikwete Cardiac Institute, Dar es Salaam, United Republic of Tanzania

${ }^{4}$ Muhimbili National Hospital, Dar es Salaam, United Republic of Tanzania

${ }^{5}$ Catholic University of Health and Allied Sciences, Mwanza, United Republic of Tanzania

${ }^{6}$ GlaxoSmithKline Research and Development, Stevenage, UK

${ }^{7}$ National Institute of Medical Research, Tanga, United Republic of Tanzania

${ }^{8}$ Cape Heart Institute Department of Medicine @ Cardiology, University of Cape

Town, Cape Town, South Africa

Acknowledgements The authors thank Bugando Medical Centre, Jakaya Kikwete Cardiac Institute and Muhimbili National Hospital's staff for collaboration in putting together the study proposal and ethical clearance.

Contributors AM and KS conceptualised the study, led the development of the study protocol, and wrote the manuscript. AM, PC, RM, BB, PK, VT, EM, EBM, AK, MJ, GK, JM, LK, JA and BPM contributed to the development of specific sections of the protocol and reviewed the manuscript. AM, PC, JM, BPM, LK, JA and KS critically reviewed both the protocol and the manuscript. All authors provided valuable input and approved the final version of the manuscript.

Funding The study is funded by the GlaxoSmithKline R\&D (Africa NonCommunicable Disease Open lab grant), grant number 8729.

Disclaimer The funder will not participate in data collection or any activity that is directly related to the execution of the research.

Competing interests JA and LK are employees and shareholders of GlaxoSmithKline. They were only involved in the review and editing of the protocol and the manuscript. They will not be involved in data collection or any activity that is directly related to the execution of the research. 
Patient and public involvement Patients and/or the public were involved in the design, or conduct, or reporting, or dissemination plans of this research. Refer to the Methods section for further details.

Patient consent for publication Not applicable.

Provenance and peer review Not commissioned; externally peer reviewed.

Open access This is an open access article distributed in accordance with the Creative Commons Attribution Non Commercial (CC BY-NC 4.0) license, which permits others to distribute, remix, adapt, build upon this work non-commercially, and license their derivative works on different terms, provided the original work is properly cited, appropriate credit is given, any changes made indicated, and the use is non-commercial. See: http://creativecommons.org/licenses/by-nc/4.0/.

ORCID iD

Pilly Chillo http://orcid.org/0000-0001-7073-1122

\section{REFERENCES}

1 Sliwa K, Libhaber E, Elliott C, et al. Spectrum of cardiac disease in maternity in a low-resource cohort in South Africa. Heart 2014;100:1967-74. doi:10.1136/heartjnl-2014-306199

2 Roos-Hesselink JW, Ruys TPE, Stein JI, et al. Outcome of pregnancy in patients with structural or ischaemic heart disease: results of a Registry of the European Society of cardiology. Eur Heart $J$ 2013:34:657-65.

3 Siu SC, Sermer M, Colman JM, et al. Prospective multicenter study of pregnancy outcomes in women with heart disease. Circulation 2001;104:515-21.

4 Regitz-Zagrosek V, Roos-Hesselink JW, Bauersachs J, et al. 2018 ESC guidelines for the management of cardiovascular diseases during pregnancy. Eur Heart $J$ 2018;39:3165-241.

5 Ashrafi R, Curtis SL. Heart disease and pregnancy. Cardiol Ther 2017:6:157-73.

6 Elliott C, Sliwa K, Burton R. Pregnancy and cardiac disease. S Afr Med J 2014;104:641.

7 Adam K. Pregnancy in women with cardiovascular diseases. Methodist Debakey Cardiovasc J 2017;13:209.

8 Khairy P, lonescu-Ittu R, Mackie AS, et al. Changing mortality in congenital heart disease. J Am Coll Cardiol 2010;56:1149-57.

9 Knight M, Bunch $\mathrm{K}$, Tuffnell $\mathrm{D}$, eds. on behalf of MBRRACE-UK. Saving Lives, Improving Mothers' Care - Lessons learned to inform maternity care from the UK and Ireland Confidential Enquiries into Maternal Deaths and Morbidity 2015-17. Oxford: National Perinatal Epidemiology Unit, University of Oxford, 2019.

10 Sliwa K, Anthony J. Late maternal deaths: a neglected responsibility. Lancet 2016:387:2072-3.

11 Sliwa K. The heart of Africa: succeeding against the odds. Lancet 2016;388:e28-36.

12 Soma-Pillay, P, Seabe J, Sliwa K. The importance of cardiovascular pathology contributing to maternal death: confidential enquiry into maternal deaths in South Africa, 2011-2013. Cardiovasc J Afr 2016;27:60-5.

13 WHO. Sustainable development goals, 2020. Available: https://www. who.int/health-topics/sustainable-development-goals [Accessed 27 Sep 2020].

14 2000-2015, Millennium Development Goals - UN Documentation: Development - Research Guides at United Nations Dag
Hammarskjöld Library, 2000. Available: https://research.un.org/en/ docs/dev/2000-2015 [Accessed 27 Sep 2020].

15 Sliwa K, Baris L, Sinning C, et al. Pregnant Women With Uncorrected Congenital Heart Disease: Heart Failure and Mortality. JACC Heart Fail 2020;8:100-10.

16 Tutarel O, Baris L, Budts W, et al. Pregnancy outcomes in women with a systemic right ventricle and transposition of the great arteries results from the ESC-EORP registry of pregnancy and cardiac disease (ROPAC). Heart 2021. doi:10.1136/heartjnl-2020-318685. [Epub ahead of print: 28 Apr 2021].

17 Ramlakhan KP, Tobler D, Greutmann M, et al. Pregnancy outcomes in women with aortic coarctation. Heart 2020. doi:10.1136/ heartjnl-2020-317513. [Epub ahead of print: 29 Oct 2020].

18 Baris L, Hakeem A, Moe T, et al. Acute coronary syndrome and ischemic heart disease in pregnancy: data from the EURObservational research Programme-European Society of cardiology registry of pregnancy and cardiac disease. J Am Heart Assoc 2020;9:e015490.

19 Khairy P, Ouyang DW, Fernandes SM, et al. Pregnancy outcomes in women with congenital heart disease. Circulation 2006;113:517-24.

20 Ford AA, Wylie BJ, Waksmonski CA, et al. Maternal congenital cardiac disease: outcomes of pregnancy in a single tertiary care center. Obstet Gynecol 2008;112:828-33.

21 Warrick CM, Hart JE, Lynch AM, et al. Prevalence and descriptive analysis of congenital heart disease in parturients: obstetric, neonatal, and anesthetic outcomes. J Clin Anesth 2015;27:492-8.

22 Koutrolou-Sotiropoulou P, Parikh PB, Miller C, et al. Impact of heart disease on maternal and fetal outcomes in pregnant women. $A m \mathrm{~J}$ Cardiol 2015;116:474-80.

23 Avila WS, Rossi EG, Ramires JAF, et al. Pregnancy in patients with heart disease: experience with 1,000 cases. Clin Cardiol 2003;26:135-42.

24 Drenthen W, Pieper PG, Roos-Hesselink JW, et al. Outcome of pregnancy in women with congenital heart disease: a literature review. J Am Coll Cardiol 2007;49:2303-11.

25 Campanharo FF, Cecatti JG, Haddad SM, et al. The impact of cardiac diseases during pregnancy on severe maternal morbidity and mortality in Brazil. PLoS One 2015;10:e0144385.

26 Rezk M, Gamal A. Maternal and fetal outcome in women with rheumatic heart disease: a 3-year observational study. Arch Gynecol Obstet 2016;294:273-8.

27 Sartain JB, Anderson NL, Barry JJ, et al. Rheumatic heart disease in pregnancy: cardiac and obstetric outcomes. Intern Med J 2012;42:978-84.

28 Lin J-H, Ling W-W, Liang A-J. [Pregnancy outcome in women with rheumatic heart disease]. Zhonghua Fu Chan Ke Za Zhi 2007;42:315-9.

29 Sliwa K, Azibani F, Baard J, et al. Reducing late maternal death due to cardiovascular disease - a pragmatic pilot study. Int $\mathrm{J}$ Cardiol 2018;272:70-6.

30 Ponikowski P, Voors AA, Anker SD, et al. 2016 ESC Guidelines for the diagnosis and treatment of acute and chronic heart failure: the task force for the diagnosis and treatment of acute and chronic heart failure of the European Society of Cardiology (ESC)Developed with the special contribution of the Heart Failure Association (HFA) of the ESC. Eur Heart J 2016;37:2129-200. doi:10.1093/eurheartj/ehw128

31 Pieske B, Tschöpe C, de Boer RA, et al. How to diagnose heart failure with preserved ejection fraction: the HFA-PEFF diagnostic algorithm: a consensus recommendation from the heart failure association (HFA) of the European Society of cardiology (ESC). Eur $J$ Heart Fail 2020;22:391-412. 